\title{
A CLASS OF CONFORMAL METRICS
}

\author{
BY MAURICE HEINS
}

Communicated by J. L. Doob, April 24, 1961

1. Conformal metrics of curvature no greater than -4 are important in certain function-theoretic questions. Such metrics were introduced by Ahlfors [1] in his paper in which it was shown that the Bloch constant was at least as large as $3^{1 / 2} / 4$. Ideas of this paper were applied to the Picard theorem and allied questions by R. M. Robinson [3]. The present communication summarizes the investigations of the author in which it is shown that the differential conditions appearing in Ahlfors's work may be replaced by conditions involving upper semi-continuity and mean-value properties. In this recasting the theory parallels that of subharmonic functions. The methods of Perron are available and serve as a basis for existence theorems for conformal metrics of constant curvature -4 . The results developed in the present investigations have a number of functiontheoretic consequences. We cite two. The first is that the Bloch constant exceeds $3^{1 / 2} / 4$. The second is the following theorem:

Suppose that $B$ is the class of nonconstant bounded analytic functions $f$ on $\{|z|<1\}$ and that $n_{1}(r ; f)$ denotes the number of zeros of $f^{\prime}$, counted according to multiplicity, in $\{|z|<r\}, 0<r<1$. Then we have

$$
\sup _{f \in B}\left\{\limsup _{r \rightarrow 1} \frac{\int_{r_{0}}^{r} t^{-1} n_{1}(t ; f) d t}{-\log (1-r)}\right\}=1, \quad 0<r_{0}<1 .
$$

2. S-K metrics. Let $F$ be a Riemann surface and let $S$ denote the family of uniformizers defining the conformal structure on $F$. By a conformal metric on $F$ we mean a function $\lambda$ with domain $S$ assigning to $\sigma \in S$ a non-negative finite-valued function $\lambda_{\sigma}$ whose domain is that of $\sigma$ such that, if $\sigma, \tau \in S$ have images with nonempty intersection $O$ and $\theta=\left\{\left(\sigma^{-1}(p), \tau^{-1}(p)\right) \mid p \in O\right\}$, then $\lambda_{\sigma}(z)=\lambda_{\tau}[\theta(z)]\left|\theta^{\prime}(z)\right|$, $z \in$ domain of $\theta$. If $\phi$ is a uniformizer, there exists a unique extension of $\lambda$ to $S \cup\{\phi\}$ which is a conformal metric in the sense of the conformal structure $S \cup\{\phi\}$. We let $\lambda_{\phi}$ denote the image of $\phi$ with respect to $\lambda$ so extended. The notion of upper semi-continuity of $\lambda$ at a point $p \in F$ is referred to that of $\lambda_{\sigma}(p \in$ image of $\sigma)$ at the point $\sigma^{-1}(p)$. Similar remarks hold for differentiability properties, inequality, and vanishing. By an $S$-K metric is meant a conformal metric on $F$ which is upper semi-continuous at each point of $F$ and satisfies the condition 
that for each $p \in F$ there exists a uniformizer $\phi, \phi(0)=p$, such that with $u=\log \lambda_{\phi}$, the circumferential mean

$$
m(r ; u)=\frac{1}{2 \pi} \int_{0}^{2 \pi} u\left(r e^{i 0}\right) d \theta
$$

satisfies

$$
\liminf _{r \rightarrow 0} r^{-2}[m(r ; u)-u(0)] \geqq\left[\lambda_{\phi}(0)\right]^{2}
$$

when $\lambda_{\phi}(0)>0$.

A conformal metric of constant curvature -4 is a nonvanishing $C^{\prime \prime}$ conformal metric $\lambda$ such that $\log \lambda_{\sigma}$ satisfies the differential equation $\Delta u=4 e^{2 u}, \sigma \in S$. The definition of an S-K metric on a region $\Omega \subset F$ is readily supplied as are the notions of the restriction of a conformal metric on $F$ to a set $E \subset F$ and of a conformal metric on $E$.

We have

THEOREM 1. Let $\lambda$ denote an $S-K$ metric on a relatively compact region $\Omega$ of a Riemann surface. Let $\mu$ denote a conformal metric of constant curvature -4 on $\Omega$. If

$$
\lim \sup \lambda / \mu \leqq 1, \quad p \in f r \Omega,
$$

then $\lambda \leqq \mu$. Here $\lambda / \mu$ is the function defined on $\Omega$ as the union of quotients $\lambda_{\sigma} \circ \sigma^{-1} / \mu_{\sigma} \circ \sigma^{-1}$. The possibility that fr $\Omega$ may be emply is allowed.

Suppose that $\lambda$ is an upper semi-continuous conformal metric on $F$ which has the property that for each $p \in F$ there exists a relatively compact neighborhood $V$ of $p$ such that whenever $\mu$ is a metric of constant curvature -4 on a region $\Omega, p \in \Omega \subset V$, and $\mu$ satisfies

$$
\lim \sup \lambda^{\Omega} / \mu \leqq 1, \quad p \in f r \Omega,
$$

$\lambda^{\Omega}$ being the restriction of $\lambda$ to $\Omega$, then $\lambda^{\Omega} \leqq \mu$. Then $\lambda$ is an S-K metric. A consequence of this result is that, if $\lambda$ is an upper semi-continuous conformal metric satisfying (2.1) with "lim sup" replacing "lim inf," then $\lambda$ is an S-K metric.

If $\lambda$ is an $\mathrm{S}-\mathrm{K}$ metric and $\mu$ is a conformal metric of constant curvature -4 on $F$ and $\lambda \leqq \mu$, then either $\lambda=\mu$ or $\lambda<\mu$.

3. Induced metric. Let $f$ denote a conformal map of a Riemann surface $F$ into a Riemann surface $G$ and let $\lambda$ denote a conformal metric on $G$. Then there exists a unique conformal metric $\mu$ on $F$ such that, whenever $\sigma$ and $\tau$ are uniformizers for $F$ and $G$ respectively for which the image of $f \circ \sigma$ is contained in the image of $\tau, \mu_{\sigma}=\lambda_{\tau} \circ \theta\left|\theta^{\prime}\right|$ where $\theta=\tau^{-1} \circ(f \circ \sigma)$. We denote $\mu$ by $[\lambda, f]$ and term it the conformal 
metric induced from $\lambda$ by $f$. If $\lambda$ is $\mathrm{S}-\mathrm{K}$, then so is $[\lambda, f]$. If $\phi$ is a conformal universal covering of a Riemann surface $F$ with domain $\{|z|<1\}$, then there exists a unique conformal metric $\gamma$ on $F$ such that $[\gamma, \phi]$ is the hyperbolic metric $\left(1-|z|^{2}\right)^{-1}|d z| . \gamma$ has constant curvature -4 and is the upper envelope of the family of S-K metrics on $F$.

4. Properties of S-K metrics. S-K metrics enjoy a number of simple properties that are useful. We list the following: (a) If $\lambda_{1}$ and $\lambda_{2}$ are S-K metrics on $F$, so is $\max \left\{\lambda_{1}, \lambda_{2}\right\}$. (b) If $\lambda$ is an $S-K$ metric and $s$ is a negative subharmonic function on $F$, then $(\exp \circ s) \lambda$ is an S-K metric on $F$. (c) If $\lambda_{1}, \cdots, \lambda_{n}$ are S-K metrics on $F$ and $\eta_{1}, \cdots, \eta_{n}$ are positive numbers, $\sum \eta_{k}=1$, then $\left(\lambda_{1}\right)^{\eta_{1}} \cdots\left(\lambda_{n}\right)^{\eta_{n}}$ is also an S-K metric on $F$. The meanings of $(\exp \circ s) \lambda$ and $\left(\lambda_{1}\right)^{\eta_{1}} \cdots\left(\lambda_{n}\right)^{\eta_{n}}$ are those given by reference to uniformizers $\sigma \in S$.

5. Perron families of S-K metrics. Let $\lambda$ denote an S-K metric on $F$ and let $\phi$ denote a uniformizer whose domain contains 0 . Let $r>0$ be such that $\{|z| \leqq r\}$ is contained in the domain of $\phi$. Then there is at most one S-K metric $\mu$ on $F$ whose restriction to $\phi(\{|z|<r\})$ is of constant curvature -4 and whose restriction to the complement of $\phi(\{|z|<r\})$ is the same as the restriction of $\lambda$ to the complement of $\phi(\{|z|<r\})$. Such an S-K metric $\mu$ is termed the $(\phi, r)$-modification of $\lambda$. An S-K metric $\nu$ on $F$ is termed a modification of $\lambda$ provided that it is the $(\phi, r)$-modification of $\lambda$ for some $(\phi, r)$. Given $\phi$, the $(\phi, r)$-modification of $\lambda(\not \equiv 0)$ exists for $r \leqq r_{0}, r_{0}$ independent of $\lambda$. [The restriction on $r$ may be omitted. However the restricted statement admits a simple proof and is adequate for the developments, which imply the unrestricted statement.]

A family $\Phi$ of S-K metrics on $F$ is termed a Perron family provided that (1) if $\lambda_{1}, \lambda_{2} \in \Phi$, then $\max \left\{\lambda_{1}, \lambda_{2}\right\} \in \Phi$; (2) if $\lambda \in \Phi$ and $\lambda \neq 0$, then each modification of $\lambda$ also belongs to $\Phi$. The basic theorem is

Theorem 2. If $\Phi$ is a Perron family containing members $\not \equiv 0$, then the upper envelope of $\Phi$ is a conformal metric of constant curvature -4 .

Theorem 2 has many applications. For example, it permits us to show that if $\Omega$ is a relatively compact Jordan region in a Riemann surface, there exists a continuous conformal metric on $\bar{\Omega}$ whose restriction to $f r \Omega$ is an assigned continuous conformal metric on $f r \Omega$ and whose restriction to $\Omega$ is a conformal metric of constant curvature -4 . Another application shows that if a Riemann surface $F$ admits a conformal universal covering with domain $\{|z|<1\}$, then for each point $a \in F$ there exists a conformal metric of constant curvature -4 on $F-\{a\}$ which vanishes at $a$. 
The method of Perron families also yields a treatment of the problem of Schwarz and Picard concerning the existence of a conformal metric of constant negative curvature [which may be normalized to be -4 ] on a compact Riemann surface $F$ less a finite number of points at which the limiting behavior of the metric is assigned. The work of Picard [2] gives a condition that is sufficient but not necessary in the case of a Riemann surface of positive genus. A necessary and sufficient condition on the assigned indices at the singular points for the problem of Schwarz and Picard to have an affirmative solution is that their sum exceed the negative of the Euler characteristic of $F$, the necessity being an immediate consequence of the formula giving the area associated with the given conformal metric in terms of the assigned indices and the Euler characteristic.

6. Bounded analytic functions on $\{|z|<1\}$. If $\gamma$ denotes the hyperbolic metric of $\{|z|<1\}$, then $[\gamma, f]$ has constant curvature -4 on $\{|z|<1\}$ less the points where $f^{\prime}$ vanishes, $f$ being a nonconstant analytic function of modulus less than one on $\{|z|<1\}$. Further $[\gamma, f]$ vanishes to the order $n(z ; f)-1$ at each point $z$ where $f^{\prime}$ vanishes, $n(z ; f)$ being the multiplicity of $f$ at $z$. In the opposite direction we have

THEOREM 3. If there exists a conformal metric $\lambda$ of constant curvature -4 on $\{|z|<1\}-E$ where $E$ is a discrete subset of $\{|z|<1\}$ such that $\lambda$ has a zero of positive integral order $\nu(a)$ at $a \in E$, then there exists a nonconstant analytic function $f$ of modulus less than one on $\{|z|<1\}$ such that the zero set of $f^{\prime}$ is $E$ and $n(a ; f)=\nu(a)+1, a \in E$.

With the aid of Theorem 3 we conclude that if $f$ is a Lindelöfian map of $\{|z|<1\}$ into a Riemann surface $G$ [or equivalently, if $f$ is a conformal map of $\{|z|<1\}$ into $G$ having a bounded Nevanlinna characteristic function], then there exists a nonconstant bounded analytic function $b$ on $\{|z|<1\}$ such that $n(z ; b)=n(z ; f),|z|<1$. Theorem 3 together with the results of the Schwarz-Picard problem for compact Riemann surfaces of positive genus yield the result that the left-hand side of (1.1) is at least at large as 1 . The inequality in the other sense follows from the Schwarz-Pick inequality.

\section{BIBLIOGRAPHY}

1. L. V. Ahlfors, An extension of Schwarz's lemma, Trans. Amer. Math. Soc. vol. 43 (1938) pp. 359-364.

2. E. Picard, Traité d'analyse, vol. 3, Paris, Gauthier-Villars, 1928.

3. R. M. Robinson, $A$ generalization of Picard's and related theorems, Duke Math. J. vol. 5 (1939) pp. 118-131.

UNIVERSITY OF ILLINOIS 